\title{
Fault Detection for Non-Gaussian Stochastic Systems with Time-Varying Delay
}

\author{
Tao Li, ${ }^{1,2}$ Kai Zhang, ${ }^{1}$ and Bo-Chao Zheng ${ }^{1}$ \\ ${ }^{1}$ School of Information and Control, Nanjing University of Information Science and Technology, Nanjing, Jiangsu 210044, China \\ ${ }^{2}$ Department of Electrical and Computer Engineering, University of Alberta, Edmonton, AB, Canada T6G 2V4
}

Correspondence should be addressed to Tao Li; litaojia@nuist.edu.cn

Received 21 April 2013; Accepted 25 May 2013

Academic Editor: Xiaojie Su

Copyright (c) 2013 Tao Li et al. This is an open access article distributed under the Creative Commons Attribution License, which permits unrestricted use, distribution, and reproduction in any medium, provided the original work is properly cited.

Fault detection (FD) for non-Gaussian stochastic systems with time-varying delay is studied. The available information for the addressed problem is the input and the measured output probability density functions (PDFs) of the system. In this framework, firstly, by constructing an augmented Lyapunov functional, which involves some slack variables and a tuning parameter, a delaydependent condition for the existence of FD observer is derived in terms of linear matrix inequality (LMI) and the fault can be detected through a threshold. Secondly, in order to improve the detection sensitivity performance, the optimal algorithm is applied to minimize the threshold value. Finally, paper-making process example is given to demonstrate the applicability of the proposed approach.

\section{Introduction}

Fault detection for control systems has been of interest for many researchers during the past three decades (see [1-7] for surveys). Effective methodologies mainly include the filter(or observer-) based approaches, the identification-based schemes, and the statistic approaches. However, most of the FD methodologies for stochastic systems only considered Gaussian systems. For example, the random process has been considered as Markov and Wiener processes in [8], respectively.

It has been shown that either the system variables are not Gaussian in $[9,10]$, existing methods may not be sufficient to characterize the non-Gaussian system behavior. Typical examples include fibre length distribution control in paper making, molecular weight distribution control, particle size distribution control in polymerization, and powder industries [8]. For such practical control problems, a new group of strategies that control the shape of PDFs for stochastic systems have been developed in the past few years (see [11, $12]$ ), where the purpose is to design a controller so that the
PDF of the system output can track a prespecified desired $\mathrm{PDF}$, as close as possible.

To simplify system modeling, B-spline neural networks had been initially used to approximate the output $\operatorname{PDF}[9,13]$. The motivation of FD via the output PDFs from the retention system in paper making was first studied in [10], where the weight dynamical system was supposed to be a precise linear model. However, linear mappings cannot change the shape of output PDFs, which implies that the fault cannot be detected through the shape change of the PDFs. To meet the requirement in complex processes, nonlinearity should be considered in the weighting dynamic behavior. Recently, a kind of observer-based FD algorithm has been established in [14], where the nonlinear weighting system was considered. However, only the uniform boundedness of the estimation error could be guaranteed in [14], which leads to some conservative criteria.

On the other hand, time delay exists commonly in dynamic systems and is frequently a source of instability and poor performance [15-17]; many works had been done about time-delay systems along the development of stochastic 
theories [18]. Recently, FD problem has been studied for timedelay stochastic systems using PDF in [19-21]. But the criteria [19-21] are only available to systems with constant delay. Meanwhile, it is noted that in practice, time-varying delay is often encountered in dynamic systems. However, up to our knowledge, there have been few results in the literature of an investigation for the FD algorithm of dynamic systems with time-varying delay by using PDF.

In this paper, we provide a further contribution to FD for non-Gaussian stochastic systems with time-varying delay based on the method in [20,21]. Firstly, by constructing an augmented Lyapunov functional, which involves some slack variables and a tuning parameter, a delay-dependent condition for the existence of FD observer is derived in terms of linear matrix inequality (LMI) and the fault can be detected through a threshold. Secondly, in order to improve the detection sensitivity performance, the optimal algorithm is applied to minimize the threshold value. Finally, paper-making process example is given to demonstrate the applicability of the proposed approach.

Notation. Throughout this paper, $R^{n}$ denotes the $n$ dimensional Euclidean space. The superscripts " $\tau$ " and "-1" stand for matrix transposition and matrix inverse, respectively; $P>(\geq 0)$ means that $P$ is real symmetric and positive definite (semidefinite). In symmetric block matrices or complex matrix expressions, $\operatorname{diag}\{\cdots\}$ stands for a block-diagonal matrix, and $*$ represents a term that is induced by symmetry. For a vector $\nu(t)$, its norm is given by $\|v(t)\|_{2}^{2}=\int_{0}^{\infty} v^{\top}(t) v(t) d t$. Matrices, if their dimensions are not explicitly stated, are assumed to be compatible for related algebraic operations.

\section{Problem Formulation and Preliminaries}

In this section, firstly, we briefly review square-root B-spline expansion technique presented in $[19,21]$, which is used to formulate the output PDFs with the dynamic weight and is essential in solving our FD problem.

For a dynamic stochastic system, its output PDFs is defined by $\gamma(z, u(t), F(t))$, where $u(t) \in R^{m}$ is control input and $F(t)$ is the fault vector to be detected. In [13, 19-21], the following square-root B-spline expansion model has been used to approximate $\gamma(z, u(t), F(t))$ :

$$
\sqrt{\gamma(z, u(t), F(t))}=\sum_{i=1}^{n} v_{i}(u, F) b_{i}(z),
$$

where $b_{i}(z)(i=1,2, \ldots, n)$ are prespecified basis functions defined on $[a, b]$ and $v_{i}(u(t), F(t))(i=1,2, \ldots, n)$ are the corresponding weights of such an expansion. Denote $B_{0}(z)=$ $\left[\begin{array}{llll}b_{1}(z) & b_{2}(z) & \cdots & b_{n-1}(z)\end{array}\right]^{\tau}$ and $\bar{v}(t):=\bar{v}(u(t), F(t))=$ $\left[\begin{array}{llll}v_{1} & v_{2} & \cdots & v_{n-1}\end{array}\right]^{\tau}$, and let $\Lambda_{1}=\int_{a}^{b} B_{0}(z) B_{0}^{\tau}(z) d z, \Lambda_{2}=$ $\int_{a}^{b} B_{0}^{\tau}(z) b_{n}(z) d z, \Lambda_{3}=\int_{a}^{b} b_{n}^{2}(z) d z \neq 0$, and $\Lambda_{0}=\Lambda_{1} \Lambda_{3}-$ $\Lambda_{2} \Lambda_{2}^{\tau}$. Furthermore, it can be verified that (1) can be rewritten as (see [19] for details)

$$
\sqrt{\gamma(z, u(t), F)}=B^{\tau}(z) \bar{v}(t)+h(\bar{v}(t)) b_{n}(z),
$$

where

$$
\begin{gathered}
B^{\tau}(z)=B_{0}^{\tau}(z)-\frac{\Lambda_{2}}{\Lambda_{3}} b_{n}(z), \\
h(\bar{v}(t))=\frac{\sqrt{\Lambda_{3}-\bar{v}^{\tau}(t) \Lambda_{0} \bar{v}^{\tau}(t)}}{\Lambda_{3}} .
\end{gathered}
$$

The relationship between the input and the weights related to the PDFs can be described by the following model:

$$
\begin{gathered}
\dot{x}(t)=A x(t)+A_{d} x(t-d(t))+G g(x(t)) \\
+H u(t)+J_{f}(t) \\
\bar{v}(t)=E x(t),
\end{gathered}
$$

where $x(t) \in R^{m}$ is the unmeasured state and $F(t) \in R^{m}$ is the fault to be detected. $A \in R^{m \times m}, A_{d} \in R^{m \times m}, G \in R^{m \times m}$, $H \in R^{m \times 1}, J_{f} \in R^{m \times m}$, and $E \in R^{m \times m}$ represent the known parametric matrices of the dynamic part of the weight system. In fact, these matrices can be estimated by using the scaling parameter identification algorithms in [22]. Similar model can be found in [21], However, only constant delay is considered in [21]. In this paper, the time delay $d(t)$ is a differential function, satisfying $0 \leq d(t) \leq h, \dot{d}(t) \leq \mu$, and the initial condition is defined by $x(t)=\phi(t)(-h \leq t \leq 0)$. In addition, similar to $[19,21]$, the following assumptions are needed.

Assumption 1. For any $x_{1}(t)$ and $x_{2}(t), \quad g(x(t))$ satisfies $g(0)=0$ and

$$
\left\|g\left(x_{1}(t)\right)-g\left(x_{2}(t)\right)\right\| \leq\left\|U_{2}\left(x_{1}(t)-x_{2}(t)\right)\right\|,
$$

where $U_{2}$ is a known matrix.

Assumption 2. There is a known matrix $U_{1}$; for any $\bar{v}_{1}(t)$ and $\bar{v}_{2}(t), h(\bar{v}(t))$ denoted by (3) satisfies the following condition:

$$
\left\|h\left(\bar{v}_{1}(t)\right)-h\left(\bar{v}_{2}(t)\right)\right\| \leq\left\|U_{1}\left(\bar{v}_{1}(t)-\bar{v}_{2}(t)\right)\right\|,
$$

where $\|\cdot\|$ is denoted as the Euclidean norm.

Inequalities (5) and (6) are typically required in the literature on FD for nonlinear systems, for example, [23, 24], which will help to simplify the design of algorithms later on.

Generally speaking a fault detection system consists of a residual generator, and a residual evaluator including an evaluation function and a threshold. We will consider two parts of fault detection systems by using the information of PDF in the following section.

2.1. Residual Generator. For the purpose of residual generation, we construct the following nonlinear observer:

$$
\begin{aligned}
\dot{\hat{x}}(t)= & A \widehat{x}(t)+A_{d} \widehat{x}(t-d(t))+G g(\widehat{x}(t)) \\
& +H u(t)+L \xi(t),
\end{aligned}
$$




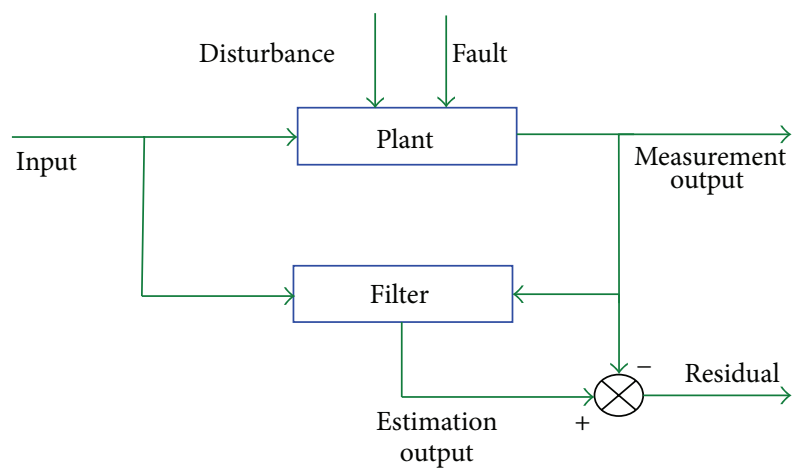

FIgURE 1: The classical residual generator.

where $\widehat{x}(t)$ is the estimated state and $L \in R^{m \times p}$ is the gain to be determined. $\xi(t)$ is the residual signal which is defined in terms of output PDFs as

$$
\begin{gathered}
\xi(t)=\int_{a}^{b} \sigma(z)(\sqrt{\gamma(z, u(t), F)}-\sqrt{\hat{\gamma}(z, u(t))}) d z, \\
\sqrt{\hat{\gamma}(z, u(t))}=B^{\tau}(z) E \hat{x}(t)+h(E \hat{x}(t)) b_{n}(z) .
\end{gathered}
$$

Remark 3. The classical residual generator design methods (such as [25-27]) are formulated in Figure 1. Different from the classical residual generator, residual $\xi(t)$ in (7) is formulated as an integral with respect to the difference of the measured PDFs and the estimated PDFs.

In order to describe the dynamics of (7), we first consider $e(t)=x(t)-\widehat{x}(t)$ and $\xi(t)$; it can be shown that

$$
\begin{aligned}
\dot{e}(t)= & \left(A-L \Gamma_{1}\right) e(t)+A_{d} e(t-d(t)) \\
& +G[g(x(t))-g(\widehat{x}(t))] \\
& -L \Gamma_{2}[h(E x(t))-h(E \widehat{x}(t))]+J_{f}(t), \\
\xi(t)= & \Gamma_{1} e(t)+\Gamma_{2}(h(E x(t))-h(E \hat{x}(t))),
\end{aligned}
$$

where

$$
\Gamma_{1}=\int_{a}^{b} \sigma(z) B^{\tau}(z) E d z, \quad \Gamma_{2}=\int_{a}^{b} \sigma(z) b_{n}(z) d z .
$$

Thus, the problem of designing an observer-based fault detection can be described as follows:

(i) design matrix $L$ such that error system (9) is asymptotically stable;

(ii) the fault can be detected by residual generator.

2.2. Residual Evaluator. After designing of FD observer, the next task for FD is the evaluation of the generated residual. One of the widely adopted approaches is to use the following logical relationship for FD:

$$
\begin{gathered}
\|r(t)\|>J_{\text {th }} \Longrightarrow \text { faults } \Longrightarrow \text { alarm }, \\
\|r(t)\| \leq J_{\text {th }} \Longrightarrow \text { no faults, }
\end{gathered}
$$

where $r(t)$ is the residual evaluation function and $J_{\text {th }}>$ 0 is the threshold. From the above logical relationship, it is clear that the threshold is important for FD sensitivity performance. In order to detect the fault more sensitively, the optimization techniques for the threshold are preferable. For this purpose, we construct a reference vector as follows:

$$
z(t)=C \xi(t)=C \Gamma_{1} e(t)+C \Gamma_{2}(h(E x(t))-h(E \widehat{x}(t))),
$$

where $C$ is a selected weighting matrix. At this stage, we can consider the suboptimal guaranteed cost problem for $J=\|z(t)\|_{2}^{2}$ subjected to error system (9) with $\widehat{x}(0)=0$ and $e(t)=\phi(t)(-h \leq t \leq 0)$. In this paper, we choose $\|z(t)\|$ and the bound $J^{*}=J^{1 / 2}$ as the residual evaluation function and threshold, respectively.

\section{Main Result}

In the section, an augmented Lyapunov-Krasovskii functional involving some slack variables and a tuning parameter is constructed and the following criterion is obtained.

Theorem 4. If for the parameters $\lambda_{i}>0(i=1,2)$ and $\varepsilon$, there exist matrices $P_{1}>0, Q_{1}>0, Q_{2}>0, T>0, P_{i}(i=$ $2, \ldots, 6)$, and $R$ such that the following inequality holds:

$$
\left[\begin{array}{ccccccccc}
\Pi_{11} & \Pi_{12} & \Pi_{13} & -P_{4}^{\tau} & P_{2}^{\tau} G & R \Gamma_{2} & -h P_{3}^{\tau} & -h P_{4}^{\tau} & \Gamma_{1}^{\tau} C^{\tau} \\
* & \Pi_{22} & \varepsilon P_{2}^{\tau} A_{d} & 0 & \varepsilon P_{2}^{\tau} G & \varepsilon R \Gamma_{2} & 0 & 0 & 0 \\
* & * & \Pi_{33} & -P_{6}^{\tau} & 0 & 0 & -h P_{5}^{\tau} & -h P_{6}^{\tau} & 0 \\
* & * & * & -Q_{2} & 0 & 0 & 0 & 0 & 0 \\
* & * & * & * & -\frac{1}{\lambda_{2}^{2}} I & 0 & 0 & 0 & 0 \\
* & * & * & * & * & -\frac{1}{\lambda_{1}^{2}} I & 0 & 0 & \Gamma_{2}^{\tau} C^{\tau} \\
* & * & * & * & * & * & -h T & 0 & 0 \\
* & * & * & * & * & * & * & -h T & 0 \\
* & * & * & * & * & * & * & * & -I
\end{array}\right]
$$

where $*$ denotes the symmetric terms in a symmetric matrix and

$$
\begin{aligned}
\Pi_{11}= & \left(P_{2}^{\tau} A-R \Gamma_{1}\right)+\left(P_{2}^{\tau} A-R \Gamma_{1}\right)^{\tau}+\frac{1}{\lambda_{1}^{2}} E^{\tau} U_{1}^{\tau} U_{1} E \\
& +\frac{1}{\lambda_{2}^{2}} U_{2}^{\tau} U_{2}+Q_{1}+Q_{2}+P_{3}^{\tau}+P_{3}, \\
\Pi_{12}= & \varepsilon A^{\tau} P_{2}-\varepsilon \Gamma_{1}^{\tau} R^{\tau}+P_{1}-P_{2}^{\tau}, \\
\Pi_{13}= & P_{2}^{\tau} A_{d}-P_{3}^{\tau}+P_{4}^{\tau}+P_{5}, \\
\Pi_{22}= & -\varepsilon P_{2}-\varepsilon P_{2}^{\tau}+h T, \\
\Pi_{33}= & -P_{5}-P_{5}^{\tau}-P_{6}-P_{6}^{\tau}-(1-\mu) Q_{1},
\end{aligned}
$$


then in the absence of $F(t)$, error system (9) with gain $L=P_{2}^{-\tau} R$ is stable and the reference vector satisfies

$$
\begin{aligned}
J= & \|z(t)\|_{2}^{2} \leq V(0):=\phi^{\tau}(0) P_{1} \phi(0) \\
& +\int_{-h}^{0} \int_{\theta}^{0} \dot{\phi}^{\tau}(s) T \dot{\phi}(s) d s d \theta \\
& +\int_{-d(0)}^{0} \phi^{\tau}(s) Q_{1} \phi(s) d s+\int_{-h}^{0} \phi^{\tau}(s) Q_{2} \phi(s) d s .
\end{aligned}
$$

Moreover, the fault can be detected when $\|z(t)\|>J^{*}=V^{1 / 2}(0)$ for $t \in[-h, \infty)$.

Proof. Define $h=h(x(t)), \widehat{h}=h(\widehat{x}(t)), g=g(x(t))$, and $\widehat{g}=g(\widehat{x}(t))$ and denote the Lyapunov function candidate as follows:

$$
V(t)=V_{1}(t)+V_{2}(t)+V_{3}(t)+V_{4}(t),
$$

where

$$
\begin{aligned}
V_{1}(t)= & \xi_{1}^{\tau}(t) M P \xi_{1}(t), \\
V_{2}(t)= & \int_{-h}^{0} \int_{t+\theta}^{t} \dot{e}^{\tau}(s) T \dot{e}(s) d s d \theta, \\
V_{3}(t)= & \int_{t-d(t)}^{t} e^{\tau}(s) Q_{1} e(s) d s+\int_{t-h}^{t} e^{\tau}(s) Q_{2} e(s) d s, \\
V_{4}(t)= & \frac{1}{\lambda_{1}^{2}} \int_{0}^{t}\left[\left\|U_{1} E e(s)\right\|^{2}-\|h-\widehat{h}\|^{2}\right] d s \\
& +\frac{1}{\lambda_{2}^{2}} \int_{0}^{t}\left[\left\|U_{2} e(s)\right\|^{2}-\|g-\hat{g}\|^{2}\right] d s
\end{aligned}
$$

with

$$
\begin{gathered}
M=\left[\begin{array}{llll}
I & 0 & 0 & 0 \\
0 & 0 & 0 & 0 \\
0 & 0 & 0 & 0
\end{array}\right], \quad P=\left[\begin{array}{ccc}
P_{1} & 0 & 0 \\
P_{2} & \varepsilon P_{2} & 0 \\
P_{3} & 0 & P_{5} \\
P_{4} & 0 & P_{6}
\end{array}\right], \\
\xi_{1}(t)=\left[\begin{array}{c}
e(t) \\
\dot{e}(t) \\
e(t-d(t))
\end{array}\right],
\end{gathered}
$$

and with $P_{1}>0, T>0, Q_{1}>0, Q_{2}>0$. It is noted that $V_{1}(t)$ is actually $e^{\tau}(t) P_{1} e(t)$. Then following (5) and (6) gives $V(t) \geq$ 0 . On the other hand, from the Leibniz-Newton formula, the following equations are true:

$$
\begin{aligned}
& \alpha_{1}:=e(t)-e(t-d(t))-\int_{t-d(t)}^{t} \dot{e}(s) d s=0, \\
& \alpha_{2}:=e(t-d(t))-e(t-h)-\int_{t-h}^{t-d(t)} \dot{e}(s) d s=0 .
\end{aligned}
$$

Hence, calculating the time derivative of $V_{1}(t)$ along the solution of (9) in the absence of $F(t)$ and using (20) yields

$$
\begin{aligned}
\dot{V}_{1}(t)= & 2 \xi_{1}(t) P^{\tau} \\
& \times\left[\begin{array}{c}
\dot{e}(t) \\
-\dot{e}(t)+\left(A-L \Gamma_{1}\right) e(t)+A_{d} e(t-d(t)) \\
+G(g-\widehat{g})-L \Gamma_{2}(h-\widehat{h}) \\
\alpha_{1} \\
\alpha_{2}
\end{array}\right] \\
= & 2 e^{\tau}(t) P_{1} \dot{e}(t)-2 e^{\tau}(t) P_{2}^{\tau} \dot{e}(t) \\
& +e^{\tau}(t)\left[\left(P_{2}^{\tau} A-R \Gamma_{1}\right)+\left(P_{2}^{\tau} A-R \Gamma_{1}\right)^{\tau}\right] e(t) \\
& +2 e^{\tau}(t) P_{2}^{\tau} A_{d} e(t-d(t))+2 e^{\tau}(t) P_{2}^{\tau} G(g-\widehat{g}) \\
& -2 e^{\tau}(t) R \Gamma_{2}(h-\widehat{h})-2 \dot{e}^{\tau}(t) P_{2}^{\tau} \dot{e}(t) \\
& +2 \varepsilon \dot{e}^{\tau}(t)\left(P_{2}^{\tau} A-R \Gamma_{1}\right) e(t) \\
& +2 \varepsilon \dot{e}^{\tau}(t) P_{2}^{\tau} A_{d}(t-d(t))+2 \varepsilon \dot{e}^{\tau}(t) P_{2}^{\tau} G(g-\widehat{g}) \\
& -2 \varepsilon \dot{e}^{\tau}(t) R \Gamma_{2}(h-\widehat{h})+2 e^{\tau}(t) P_{3}^{\tau} e(t) \\
& -2 e^{\tau}(t) P_{3}^{\tau} e(t-d(t))-2 e^{\tau}(t) P_{3}^{\tau} \int_{t-d(t)}^{t} \dot{e}(s) d s \\
& +2 e^{\tau}(t-d(t)) P_{5}^{\tau} e(t) \\
& -2 e^{\tau}(t-d(t)) P_{5}^{\tau} e(t-d(t)) \\
& \left.-2 e^{\tau}(t) \Theta_{1} \eta(t), \quad d(t)\right) P_{5}^{\tau} \int_{t-d(t)}^{t} \dot{e}(s) d s \\
& +2 e^{\tau}(t-d(t)) P_{6}^{\tau} e(t-d(t)) \\
& +2 e^{\tau}(t) P_{4}^{\tau} e(t-d(t))-2 e^{\tau}(t) P_{4}^{\tau} e(t-h) \\
& -2 e^{\tau}(t) P_{4}^{\tau} \int_{t-h}^{t-d(t)}(s) d s \\
& \\
&
\end{aligned}
$$

where

$$
\Theta_{1}=\left[\begin{array}{cccccccc}
\Theta_{11} & \Pi_{12} & \Pi_{13} & -P_{4}^{\tau} & P_{2}^{\tau} G & R \Gamma_{2} & -P_{3}^{\tau} & -P_{4}^{\tau} \\
* & \Theta_{22} & \varepsilon P_{2}^{\tau} A_{d} & 0 & \varepsilon P_{2}^{\tau} G & \varepsilon R \Gamma_{2} & 0 & 0 \\
* & * & \Theta_{33} & -P_{6}^{\tau} & 0 & 0 & -P_{5}^{\tau} & -P_{6}^{\tau} \\
* & * & * & 0 & 0 & 0 & 0 & 0 \\
* & * & * & * & 0 & 0 & 0 & 0 \\
* & * & * & * & * & 0 & 0 & 0 \\
* & * & * & * & * & * & 0 & 0 \\
* & * & * & * & * & * & * & 0
\end{array}\right]
$$




$$
\eta(t)=\left[\begin{array}{c}
e(t) \\
\dot{e}(t) \\
e(t-d(t)) \\
e(t-h) \\
(g-\widehat{g}) \\
(h-\widehat{h}) \\
\int_{t-d(t)}^{t} \dot{e} d s \\
\int_{t-h}^{t-d(t)} \dot{e} d s
\end{array}\right],
$$

where

$$
\begin{aligned}
& \Theta_{11}=\left(P_{2}^{\tau} A-R \Gamma_{1}\right)+\left(P_{2}^{\tau} A-R \Gamma_{1}\right)^{\tau}+P_{3}+P_{3}^{\tau}, \\
& \Theta_{22}=-\varepsilon P_{2}-\varepsilon P_{2}^{\tau}, \\
& \Theta_{33}=-P_{5}-P_{5}^{\tau}-P_{6}-P_{6}^{\tau},
\end{aligned}
$$

with $R=P_{2}^{\tau} L$ and $\Pi_{12}, \Pi_{13}$ being defined in (14). It follows from Jensen integral inequality with $d(t) \leq h$ that one can obtain

$$
\begin{aligned}
\dot{V}_{2}(t)= & h \dot{e}^{\tau} T \dot{e}-\int_{t-h}^{t} \dot{e}^{\tau} T \dot{e} d s \\
= & h \dot{e}^{\tau} T \dot{e}-\int_{t-h}^{t-d(t)} \dot{e}^{\tau} T \dot{e} d s-\int_{t-d(t)}^{t} \dot{e}^{\tau} T \dot{e} d s \\
\leq & h \dot{e}^{\tau}(t) T \dot{e}(t) \\
& -\frac{1}{h}\left(\int_{t-h}^{t-d(t)} \dot{e}(s) d s\right)^{\tau} T\left(\int_{t-h}^{t-d(t)} \dot{e}(s) d s\right) \\
& -\frac{1}{h}\left(\int_{t-d(t)}^{t} \dot{e}(s) d s\right)^{\tau} T\left(\int_{t-d(t)}^{t} \dot{e}(s) d s\right) .
\end{aligned}
$$

On the other hand, it can be verified that

$$
\begin{aligned}
\dot{V}_{3}(t)= & e^{\tau}(t)\left(Q_{1}+Q_{2}\right) e(t)-(1-\mu) e^{\tau}(t-d(t)) \\
& \times Q_{1} e(t-d(t))-e^{\tau}(t-h) Q_{2} e(t-h), \\
\dot{V}_{4}(t)= & \frac{1}{\lambda_{1}^{2}} E^{\tau} U_{1}^{\tau} U_{1} E+\frac{1}{\lambda_{2}^{2}} U_{2}^{\tau} U_{2} \\
& -\frac{1}{\lambda_{1}^{2}}(h-\widehat{h})^{\tau}(h-\widehat{h})-\frac{1}{\lambda_{2}^{2}}(g-\widehat{g})^{\tau}(g-\widehat{g}) .
\end{aligned}
$$

It then follows from (21)-(25) that

$$
\dot{V}(t) \leq \eta^{\tau}(t) \Xi_{0} \eta(t),
$$

where $\Xi_{0}=\Theta_{1}+\operatorname{diag}\left\{Q_{1}+Q_{2}+\left(1 / \lambda_{1}^{2}\right) E^{\tau} U_{1}^{\tau} U_{1} E+\right.$ $\left(1 / \lambda_{2}^{2}\right) U_{2}^{\tau} U_{2}, h T,-(1-\mu) Q_{1},-Q_{2},-\left(1 / \lambda_{2}^{2}\right) I,-\left(1 / \lambda_{1}^{2}\right) I,-(1 / h)$ $T,-(1 / h) T\}$. Applying the Schur complement to (14) gives $\Xi_{0}<0$, which implies $\dot{V}(t)<0$ and error system (9) is asymptotically stable.

Furthermore, we denote an auxiliary cost function as

$$
J=\int_{0}^{\infty}\left[z^{\tau}(\tau) z(\tau)+\dot{V}(\tau)\right] d \tau
$$

Note that

$$
\begin{aligned}
z^{\tau}(t) z(t)= & e^{\tau}(t) \Gamma_{1}^{\tau} C^{\tau} C \Gamma_{1} e(t)+2 e^{\tau}(t) \Gamma_{1}^{\tau} C^{\tau} C \Gamma_{2}(h-\widehat{h}) \\
& +(h-\widehat{h})^{\tau} \Gamma_{2}^{\tau} C^{\tau} C \Gamma_{2}(h-\widehat{h}) .
\end{aligned}
$$

From (14), $J<0$ holds. On the other hand, it can be shown that

$$
\begin{aligned}
J & =\int_{0}^{\infty} z^{\tau}(\tau) z(\tau) d \tau+\lim _{T \rightarrow \infty}(V(T)-V(0)) \\
& \leq\|z(t)\|^{2}-V(0)
\end{aligned}
$$

where $V(0)$ is defined in (16). Therefore (16) is satisfied. This completes the proof.

Remark 5. Theorem 4 is based on a newly proposed augmented Lyapunov functional of form (17), which contains a structure more general than the traditional ones as those in $[19,20]$ for involving $\dot{e}(t)$ and $e(t-d(t))$ in the first term of (17). In addition, the importance of augmented Lyapunov functional is that it separates Lyapunov function matrix $P_{1}$ from $A, A_{d}$; that is, there are no terms containing the product of $P_{1}$ and any of them, which makes the design of the fault detection observer more easier.

Remark 6. Recently, Li et al. [21] provided a fault detection observer design criterion by using PDFs for stochastic system with constant delay. Compared with the criterion in [21], the advantage in our paper is that time-varying delay is considered and the design criterion includes more delay information. Moreover, it is noted that setting $P_{3}=Y, P_{5}=$ $W, Q_{2}=0$, and $P_{4}=P_{6}=0$ in Theorem 4 yields precisely Theorem 1 in [21]. So, Theorem 4 in this paper is an extension of the result in [21].

In order to improve fault detection sensitivity, the following result provides an optimization algorithm in order to make the threshold $J^{*}$ smaller.

Theorem 7. Consider error system (9) with cost function (27). For given parameters $\lambda_{i}(i=1,2)$ and $\varepsilon$, if the following optimization problem:

$$
\min \left\{\alpha+\operatorname{tr}\left(\Omega_{1}\right)+\operatorname{tr}\left(\Omega_{2}\right)+\operatorname{tr}\left(\Omega_{3}\right)\right\}
$$

subject to

(i) $\operatorname{LMI}(14)$,

(ii) $\left[\begin{array}{cc}-\alpha & \phi^{\tau}(0) P_{1} \\ P_{1} \phi(0) & -P_{1}\end{array}\right]<0$,

(iii) $\left[\begin{array}{cc}-\Omega_{1} & Y_{1}^{\tau} Q_{1} \\ Q_{1} Y_{1} & -Q_{1}\end{array}\right]<0$,

(iv) $\left[\begin{array}{cc}-\Omega_{2} & Y_{2}^{\tau} Q_{2} \\ Q_{2} Y_{2} & -Q_{2}\end{array}\right]<0$,

(v) $\left[\begin{array}{cc}-\Omega_{3} & Y_{3}^{\tau} T \\ T Y_{3} & -T\end{array}\right]<0$, 
where

$$
\begin{gathered}
\int_{-d(0)}^{0} \phi(s) \phi^{\tau}(s) d s=Y_{1} Y_{1}^{\tau}, \\
\int_{-h}^{0} \phi(s) \phi^{\tau}(s) d s=Y_{2} Y_{2}^{\tau}, \\
\int_{-h}^{0} \int_{\theta}^{0} \dot{\phi}(s) \dot{\phi}^{\tau}(s) d s d \theta=Y_{3} Y_{3}^{\tau},
\end{gathered}
$$

has solutions with positive definite matrices $P_{1}, Q_{1}, Q_{2}, T, \Omega_{1}$, $\Omega_{2}$, and $\Omega_{3}$; matrices $P_{i}(i=2, \ldots, 6)$ and $R$; and positive scalar $\alpha$, then in the absence of $F(t)$, error system (9) with gain $L=P_{2}^{-\tau} R$ is stable and the smallest threshold satisfies

$$
J^{*}=\left\{\alpha+\operatorname{tr}\left(\Omega_{1}\right)+\operatorname{tr}\left(\Omega_{2}\right)+\operatorname{tr}\left(\Omega_{3}\right)\right\}^{1 / 2}
$$

for $t \in[-h, \infty)$.

Proof. By Theorem 4, (i) is clear. Also, it follows from the Schur complement that (ii), (iii), (iv), and (v) are equivalent to

$$
\begin{gathered}
\phi^{\tau}(0) P_{1} \phi(0)<\alpha, \\
Y_{1}^{\tau} Q_{1} Y_{1}<\Omega_{1}, \\
Y_{2}^{\tau} Q_{2} Y_{2}<\Omega_{2}, \\
Y_{3}^{\tau} T Y_{3}<\Omega_{3},
\end{gathered}
$$

respectively. On the other hand

$$
\begin{aligned}
\int_{-d(0)}^{0} & \phi^{\tau}(s) Q_{1} \phi(s) d s \\
& =\int_{-d(0)}^{0} \operatorname{tr}\left(\phi^{\tau}(s) Q_{1} \phi(s)\right) d s \\
& =\operatorname{tr}\left(Q_{1}^{1 / 2} \int_{-d(0)}^{0} \phi(s) \phi^{\tau}(s) d s Q_{1}^{1 / 2}\right) \\
& =\operatorname{tr}\left(Q_{1}^{1 / 2} Y_{1} Y_{1}^{\tau} Q_{1}^{1 / 2}\right)=\operatorname{tr}\left(Y_{1}^{\tau} Q_{1} Y_{1}\right)<\operatorname{tr}\left(\Omega_{1}\right) \\
\int_{-h}^{0} \phi^{\tau} & (s) Q_{2} \phi(s) d s \\
& =\int_{-h}^{0} \operatorname{tr}\left(\phi^{\tau}(s) Q_{2} \phi(s)\right) d s \\
& =\operatorname{tr}\left(Q_{2}^{1 / 2} \int_{-h}^{0} \phi(s) \phi^{\tau}(s) d s Q_{2}^{1 / 2}\right) \\
& =\operatorname{tr}\left(Q_{2}^{1 / 2} Y_{2} Y_{2}^{\tau} Q_{2}^{1 / 2}\right)=\operatorname{tr}\left(Y_{2}^{\tau} Q_{2} Y_{2}\right)<\operatorname{tr}\left(\Omega_{2}\right)
\end{aligned}
$$

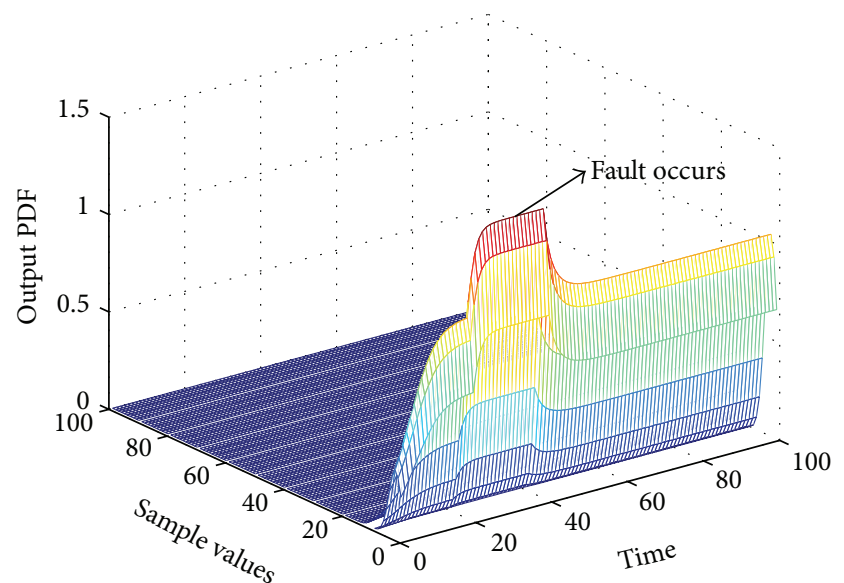

FIGURE 2: 3D mesh plot of the measured output PDFs.

$$
\begin{aligned}
\int_{h}^{0} \int_{\theta}^{0} \dot{\phi}^{\tau}(s) T \dot{\phi}(s) d s d \theta \\
\quad=\int_{h}^{0} \int_{\theta}^{0} \operatorname{tr}\left(\dot{\phi}^{\tau}(s) T \dot{\phi}(s)\right) d s d \theta \\
\quad=\operatorname{tr}\left(T^{1 / 2} \int_{h}^{0} \int_{\theta}^{0} \dot{\phi}(s) \dot{\phi}^{\tau}(s) d s d \theta T^{1 / 2}\right) \\
\quad=\operatorname{tr}\left(T^{1 / 2} Y_{3} Y_{3}^{\tau} T^{1 / 2}\right)=\operatorname{tr}\left(Y_{3}^{\tau} T Y_{3}\right)<\operatorname{tr}\left(\Omega_{3}\right)
\end{aligned}
$$

Hence, it follows from (17) that

$$
J<\alpha+\operatorname{tr}\left(\Omega_{1}\right)+\operatorname{tr}\left(\Omega_{2}\right)+\operatorname{tr}\left(\Omega_{3}\right) .
$$

Thus, the minimization of $J$ implies the minimization of the threshold values $J^{*}=J^{1 / 2}$.

\section{Simulations}

An application of paper-making process is given to demonstrate the applicability of our proposed approach. The basis functions are selected in a similar way to [20] as follows:

$$
\begin{gathered}
B(z)=\left[b_{1}(z), b_{2}(z), \ldots, b_{9}(z)\right]^{\tau}, \\
b_{i}(z)=\exp \left(-\left(z-z_{i}\right)^{2} \sigma_{i}^{-2}\right), \quad(i=1,2, \ldots, 10), \\
z_{i}=0.003+0.006(i-1), \quad \sigma_{i}=0.003, \quad(i=1,2, \ldots, 10) .
\end{gathered}
$$

Consider the following weighting system:

$$
\begin{gathered}
\dot{x}(t)=A x(t)+A_{d} x(t-d(t))+G g(x(t)) \\
+H u(t)+J_{f}(t), \\
\bar{v}(t)=x(t),
\end{gathered}
$$




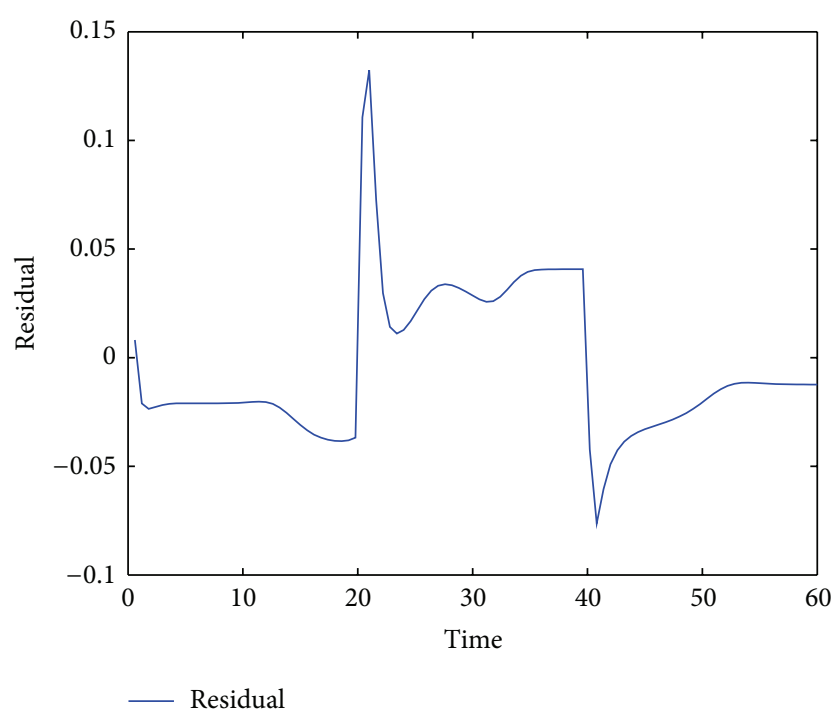

FIGURE 3: The response of residual signal.

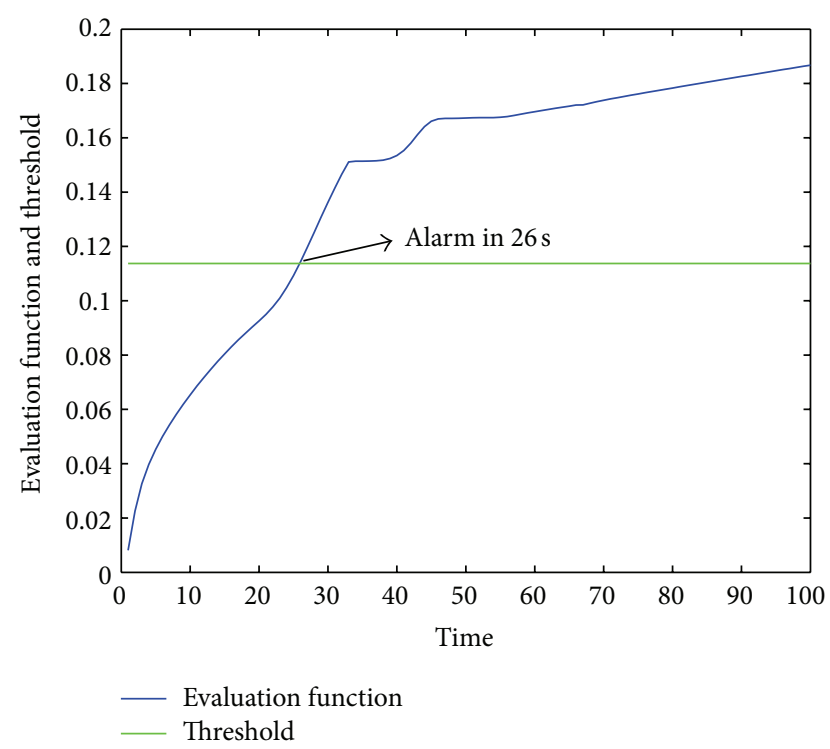

FIgURE 4: Threshold and the response of evaluation function.

where

$$
\begin{aligned}
& A=\operatorname{diag}\{-0.83,-0.83, \ldots,-0.83\} \in R^{9 \times 9}, \\
& A_{d}=\operatorname{diag}\{0.2,0.2, \ldots, 0.2\} \in R^{9 \times 9} \text {, } \\
& G=\operatorname{diag}\{1,1, \ldots, 1\} \in R^{9 \times 9}, \\
& H=\left[\begin{array}{lllllllll}
0 & 0.01 & 0.01 & 0.01 & 0.01 & 0.01 & 0.01 & 0.01 & 0
\end{array}\right]^{\tau}, \\
& J_{f}=\operatorname{diag}\{1.18,0,1.56,0,1.56,0,1.56,1.56,1.56\} \text {, }
\end{aligned}
$$

$g(x(t))=\sin (x(t))$, and $u(t)$ is random number. By setting $\sigma(z)=1$, then the following parametric matrices related to the B-spline approximation can be obtained:

$$
\begin{aligned}
& \Lambda_{0}=10^{-5} \\
& \left.\times \begin{array}{ccccccccc}
2.400 & 0.956 & 0.048 & 0 & 0 & 0 & 0 & 0 & 0 \\
0.956 & 2.607 & 0.959 & 0.047 & 0 & 0 & 0 & 0 & 0 \\
0.048 & 0.959 & 2.605 & 0.956 & 0.049 & 0.001 & 0 & 0 & 0 \\
0 & 0.047 & 0.956 & 2.601 & 0.960 & 0.048 & 0 & 0 & 0 \\
0 & 0 & 0.049 & 0.960 & 2.605 & 0.958 & 0.048 & 0.001 & 0 \\
0 & 0 & 0.001 & 0.048 & 0.958 & 2.605 & 0.960 & 0.049 & 0 \\
0 & 0 & 0 & 0 & 0.048 & 0.960 & 2.601 & 0.956 & 0.047 \\
0 & 0 & 0 & 0 & 0.001 & 0.049 & 0.956 & 2.604 & 0.939 \\
0 & 0 & 0 & 0 & 0 & 0 & 0.047 & 0.939 & 2.226
\end{array}\right],
\end{aligned}
$$$$
\Lambda_{1}=10^{-3} \times\left[\begin{array}{ccccccccc}
4.9 & 2 & 0.1 & 0 & 0 & 0 & 0 & 0 & 0 \\
2 & 5.3 & 2 & 0.1 & 0 & 0 & 0 & 0 & 0 \\
0.1 & 2 & 5.3 & 2 & 0.1 & 0 & 0 & 0 & 0 \\
0 & 0.1 & 2 & 5.3 & 2 & 0.1 & 0 & 0 & 0 \\
0 & 0 & 0.1 & 2 & 5.3 & 2 & 0.1 & 0 & 0 \\
0 & 0 & 0 & 0.1 & 2 & 5.3 & 2 & 0.1 & 0 \\
0 & 0 & 0 & 0 & 0.1 & 2 & 5.3 & 2 & 0.1 \\
0 & 0 & 0 & 0 & 0 & 0.1 & 2 & 5.3 & 2 \\
0 & 0 & 0 & 0 & 0 & 0 & 0.1 & 2 & 5.3
\end{array}\right]
$$

$\Lambda_{2}=\left[\begin{array}{llllllllll}0 & 0 & 0 & 0 & 0 & 0 & 0 & 0.0001 & 0.0020\end{array}\right]$,

$\Lambda_{3}=0.0049$.

It can be verified that $U_{1}=\operatorname{diag}\{0.05,0.05, \ldots, 0.05\} \in$ $R^{9 \times 9}$ and it can be supposed that $U_{2}=\operatorname{diag}\{1,1, \ldots, 1\} \in$ $R^{9 \times 9}$. Corresponding to (11), it can be calculated that $\Gamma_{1}=$ $10^{-2} \times\left[\begin{array}{lllllllll}6.3 & 7.5 & 7.5 & 7.5 & 7.5 & 7.5 & 7.5 & 7.4 & 5.0\end{array}\right], \Gamma_{2}=0.0063$. In this case, $d(t)=0.5 \sin (t), \mu=0.5, h=0.5$, and the initial value of observer (7) is selected as $\widehat{x}(t)=0 \in R^{9}$ for all $0 \leq t \leq$ $+\infty$, while the initial value of (4) is selected as $x_{1}(t)=2.05+$ $\exp (t-2), x_{2}(t)=1.05+\exp (t-2), x_{5}(t)=-2.05+\exp (t-2)$, $x_{9}(t)=3.0+\exp (t-2)$, and $x_{3}(t)=x_{4}(t)=x_{6}(t)=x_{7}(t)=$ $x_{8}(t)=0,(-0.5 \leq t \leq 0)$. The fault is supposed to be

$$
\begin{aligned}
& F(t)
\end{aligned}
$$

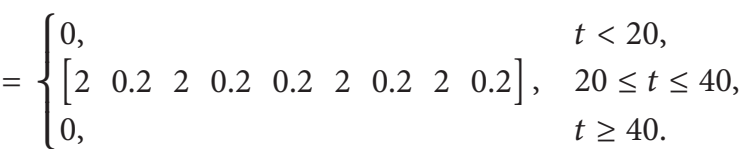

Figure 2 shows the changes of the output PDF when the fault occurs. With the detection observer, Figure 3 demonstrates the responses of the residual signal. The threshold can be computed to give $J^{*}=0.1137$ by using Theorem 7 and the residual satisfies $z(t) \geq 0.1143>J^{*}$ for $t>26$. However, it is infeasible by using Theorem 2 in [21]. Thus, a better FD algorithm may more rapidly detect the occurrence of faults. From Figure 4, we can see that the fault can be detected $6 \mathrm{~s}$ after its occurrence.

\section{Conclusion}

The FD problem is studied for a class of non-Gaussian stochastic systems with time-varying delay using augmented 
Lyapunov functional approach, where only the output PDFs can be measured rather than an output signal. A new delaydependent FD observer design criterion is obtained. And the fault can be detected with an optimal threshold, where the guaranteed cost optimization algorithm is applied to minimize it. Further work will focus on the fault diagnosis and fault tolerant for these systems.

\section{Acknowledgments}

This work is supported by the NSF of China (nos. 60904025 and 61104103) and in part by Qing Lan project of Jiang $\mathrm{Su}$ province and the scholarship from China Scholarship Council. The authors are very grateful to Professor Qing Zhao in the Department of Electrical and Computer Engineering, University of Alberta, for her helpful suggestions which have helped them in improving the quality of the paper.

\section{References}

[1] L. G. Wu, X. M. Yao, and W. X. Zheng, "Generalized $H_{2}$ fault detection for two-dimensional Markovian jump systems," Automatica, vol. 48, pp. 1741-1750, 2012.

[2] X. Y. Yao, L. G. Wu, and W. X. Zheng, "Fault detection filter design for Markovian jump singular systems with intermittent measurements," IEEE Transactions on Signal Processing, vol. 59, no. 7, pp. 3099-3109, 2011.

[3] L. G. Wu, X. J. Su, and P. Shi, "Mixed $H_{2} / H_{\infty}$ approach to fault detection of discrete linear repetitive processes," Journal of the Franklin Institute, vol. 348, no. 2, pp. 393-414, 2011.

[4] T. Li and Y. C. Zhang, "Fault detection and diagnosis for stochastic systems via output PDFs," Journal of the Franklin Institute, vol. 348, no. 6, pp. 1140-1152, 2011.

[5] T. J. Crowley, E. S. Meadows, E. Kostoulas, and F. J. Doyle III, "Control of particle size distribution described by a population balance model of semibatch emulsion polymerization," Journal of Process Control, vol. 10, no. 5, pp. 419-432, 2000.

[6] D. Zhang, H. Wang, B. Lu, and Z. Wang, "LMI-based fault detection fuzzy observer design with multiple performance constraints for a class of non-linear systems: comparative study," International Journal of Innovative Computing, Information and Control, vol. 8, no. 1, pp. 633-645, 2012.

[7] A. Benzaouia, M. Ouladsine, A. Naamane, and B. Ananou, "Fault detection for uncertain delayed switching discrete-time systems," International Journal of Innovative Computing, Information and Control, vol. 8, pp. 8049-8062, 2012.

[8] H. Wang and P. Afshar, "ILC-based fixed-structure controller design for output PDF shaping in stochastic systems using LMI techniques," IEEE Transactions on Automatic Control, vol. 54, no. 4, pp. 760-773, 2009.

[9] H. Wang, "Detecting faults in dynamic and bounded stochastic distributions: an observer based techniques," in Proceedings of the American Control Conference (ACC '01), pp. 482-487, Arlington, Va, USA, June 2001.

[10] H. Wang and W. Lin, "Applying observer based FDI techniques to detect faults in dynamic and bounded stochastic distributions," International Journal of Control, vol. 73, no. 15, pp. 14241436, 2000.

[11] M. Kárný, J. Böhm, T. V. Guy, and P. Nedoma, "Mixture-based adaptive probabilistic control," International Journal of Adaptive Control and Signal Processing, vol. 17, no. 2, pp. 119-132, 2003.
[12] H. Wang, Bounded Dynamic Stochastic Systems: Modelling and Control, Springer, London, UK, 2000.

[13] L. Guo and H. Wang, "PID controller design for output PDFs of stochastic systems using linear matrix inequalities," IEEE Transactions on Systems, Man, and Cybernetics B, vol. 35, no. 1, pp. 65-71, 2005.

[14] L. Guo and H. Wang, "Fault detection and diagnosis for general stochastic systems using B-spline expansions and nonlinear filters," IEEE Transactions on Circuits and Systems I, vol. 52, no. 8, pp. 1644-1652, 2005.

[15] G. J. Zhang, C. S. Han, Y. Guan, and L. G. Wu, "Exponential stability analysis and stabilization of discrete-time nonlinear switched systems with time delays," International Journal of Innovative Computing, Information and Control, vol. 8, no. 3, pp. 1973-1986, 2012.

[16] X. Su, P. Shi, L. G. Wu, and Y. D. Song, "A novel control design on discrete-time Takagi-Sugeno fuzzy systems with time-varying delays," IEEE Transactions on Fuzzy Systems, 2012.

[17] X. Su, L. G. Wu, and P. Shi, "Senor networks with random link failures: distributed filtering for T-S fuzzy systems," IEEE Transactions on Industrial Informatics, 2012.

[18] X. Su, L. G. Wu, P. Shi, and Y. D. Song, " $H_{\infty}$ model reduction of T-S fuzzy stochastic systems," IEEE Transactions on Systems, Man, and Cybernetics B, vol. 42, pp. 1574-1585, 2012.

[19] L. Guo, Y.-M. Zhang, H. Wang, and J.-C. Fang, “Observer-based optimal fault detection and diagnosis using conditional probability distributions," IEEE Transactions on Signal Processing, vol. 54, no. 10, pp. 3712-3719, 2006.

[20] Y. M. Zhang, L. Guo, and H. Wang, "Filter-based fault detection and diagnosis using output PDFs for stochastic systems with time delays," International Journal of Adaptive Control and Signal Processing, vol. 20, no. 4, pp. 175-194, 2006.

[21] T. Li, L. Guo, and L. Y. Wu, "Observer-based optimal fault detection using PDFs for time-delay stochastic systems," Nonlinear Analysis: Real World Applications, vol. 9, no. 5, pp. 2337-2349, 2008.

[22] H. Yue and H. Wang, "Recent developments in stochastic distribution control: a review," Measurement and Control, vol. 36, no. 7, pp. 209-215, 2003.

[23] B. Jiang and F. N. Chowdhury, "Fault estimation and accommodation for linear MIMO discrete time Systems," IEEE Transactions on Control Systems Technology, vol. 13, pp. 493-499, 2005.

[24] B. Jiang and F. N. Chowdhury, "Parameter fault detection and estimation of a class of nonlinear systems using observers," Journal of the Franklin Institute, vol. 342, no. 7, pp. 725-736, 2005.

[25] J. Liu, J. L. Wang, and G.-H. Yang, "Reliable guaranteed variance filtering against sensor failures," IEEE Transactions on Signal Processing, vol. 51, no. 5, pp. 1403-1411, 2003.

[26] Q. Zhao and Z. Xu, "Design of a novel knowledge-based fault detection and isolation scheme," IEEE Transactions on Systems, Man, and Cybernetics B, vol. 34, no. 2, pp. 1089-1095, 2004.

[27] M. Zhong, S. X. Ding, J. Lam, and H. Wang, "An LMI approach to design robust fault detection filter for uncertain LTI systems," Automatica, vol. 39, no. 3, pp. 543-550, 2003. 


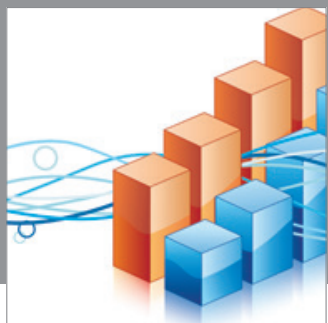

Advances in

Operations Research

mansans

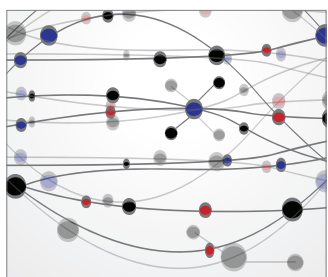

The Scientific World Journal
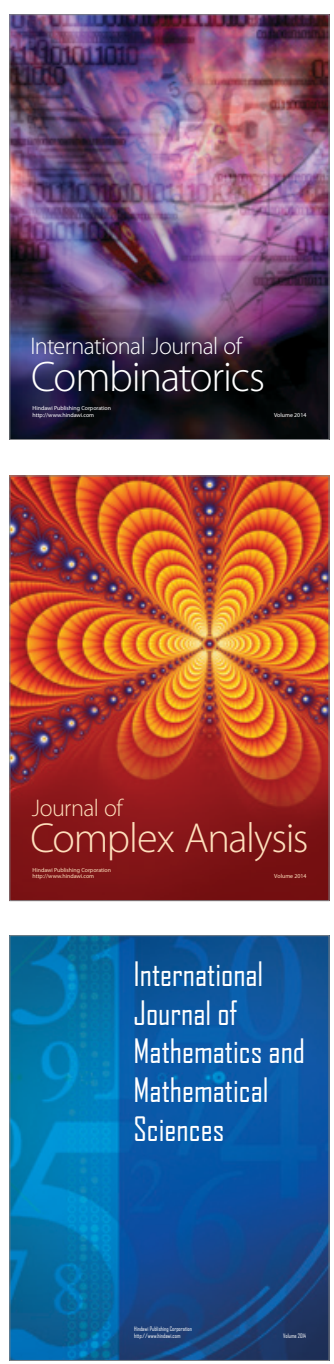
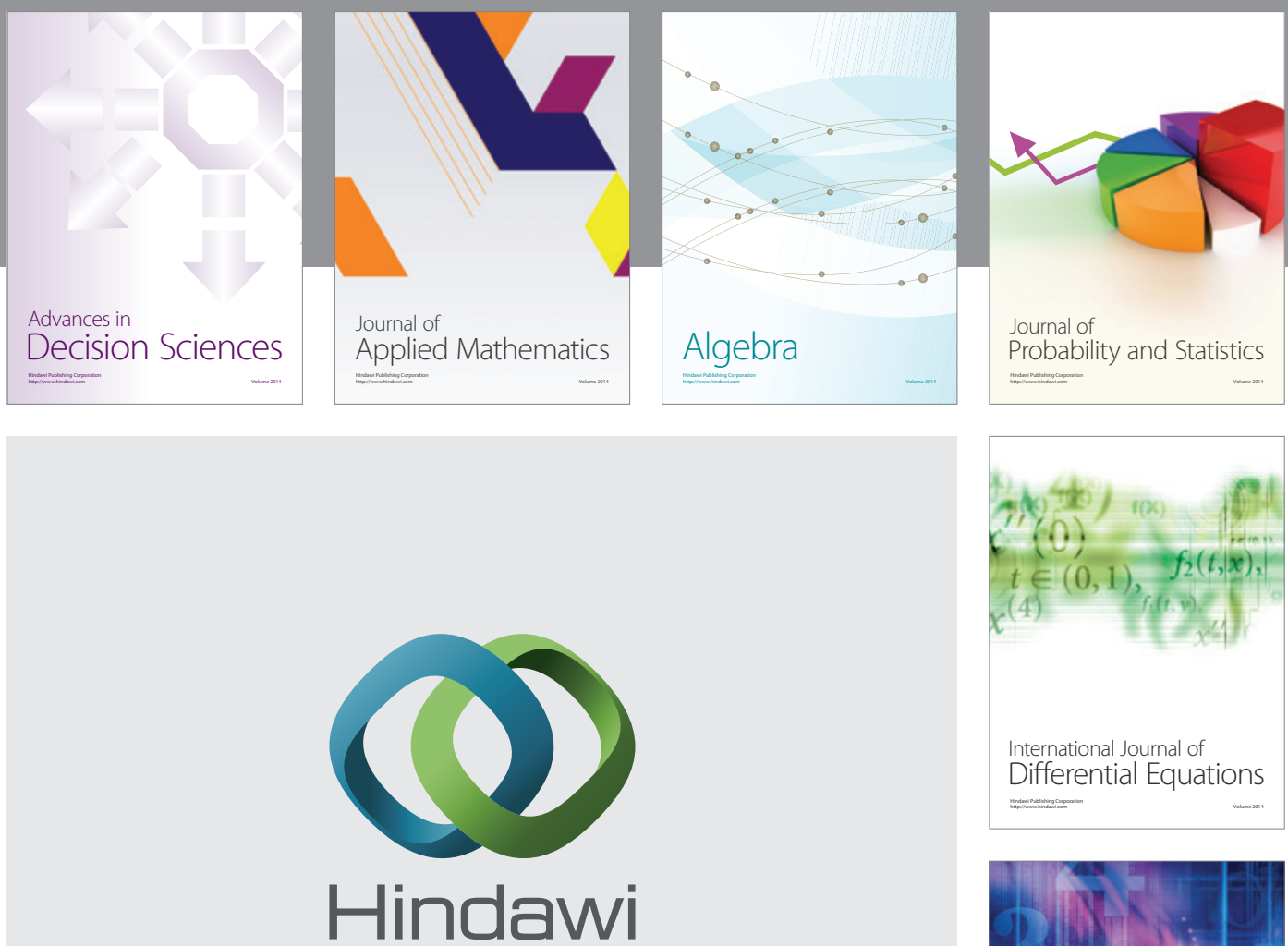

Submit your manuscripts at http://www.hindawi.com
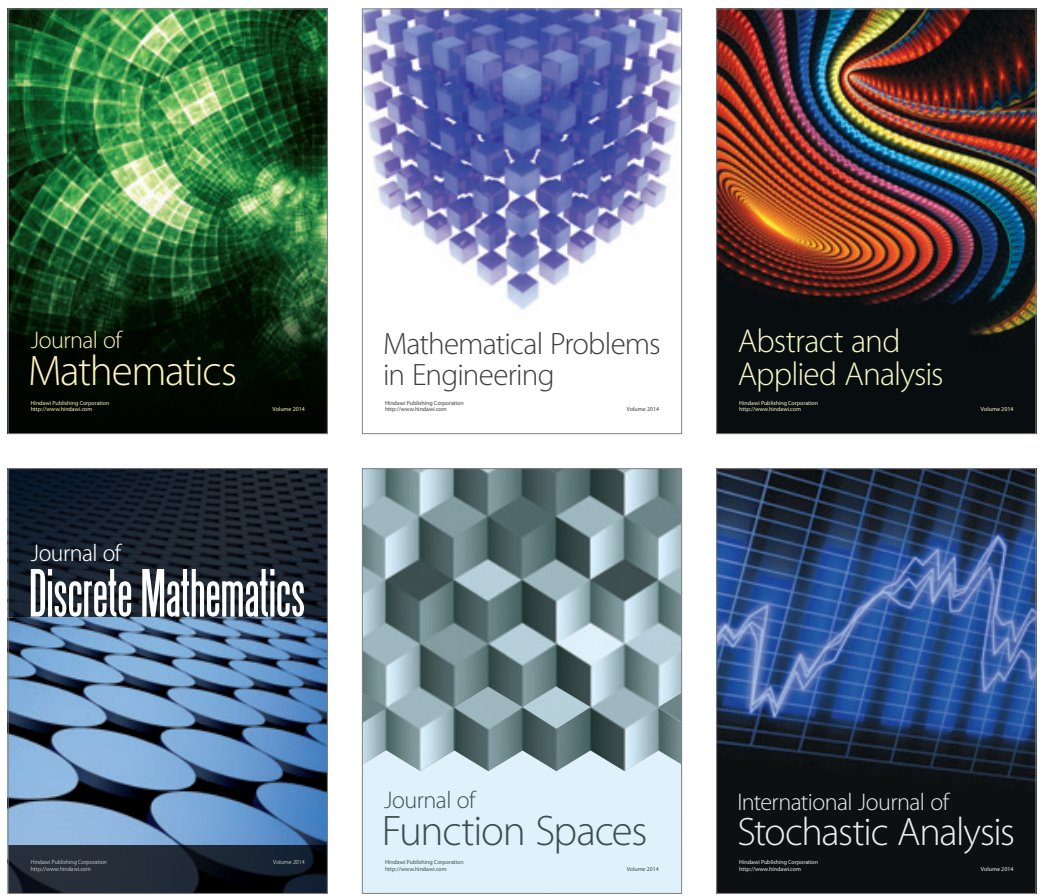

Journal of

Function Spaces

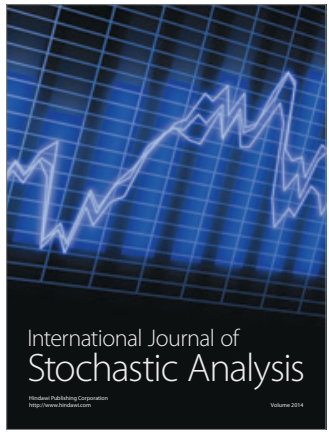

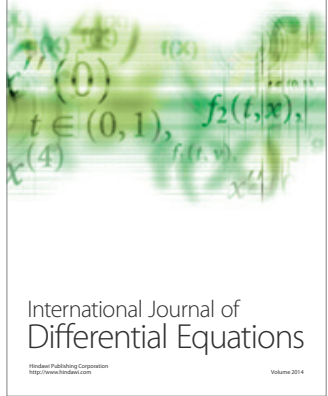
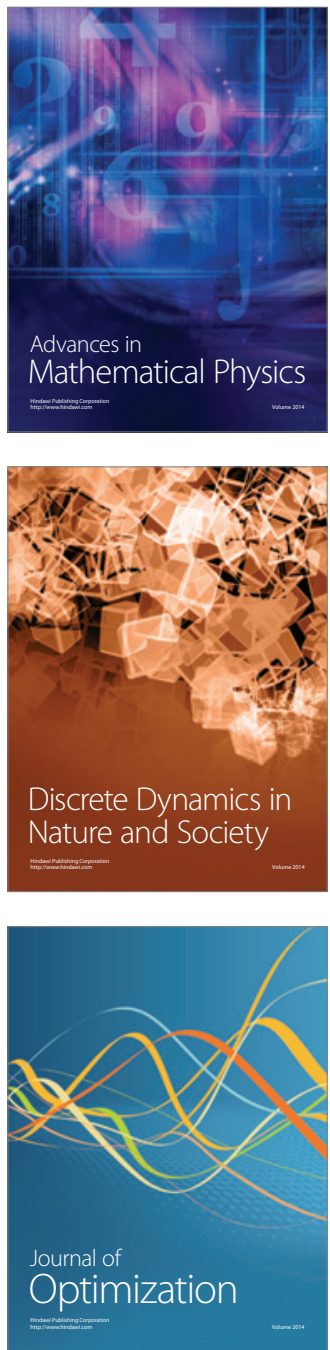